\title{
Application of Gradient Projection for Sparse Reconstruction to Compressed Sensing for Image Reconstruction of Electrical Capacitance Tomography
}

\author{
Lifeng Zhang*, Yajie Song \\ Department of Automation, North China Electric Power University, Baoding, China \\ Email address: \\ hdlfzhang a 126.com (Lifeng Zhang),ncepu_songyajie $a$ foxmail.com (Yajie Song) \\ ${ }^{*}$ Corresponding author
}

To cite this article:

Lifeng Zhang, Yajie Song. Application of Gradient Projection for Sparse Reconstruction to Compressed Sensing for Image Reconstruction of Electrical Capacitance Tomography. Journal of Electrical and Electronic Engineering. Vol. 6, No. 2, 2018, pp. 46-52. doi: $10.11648 /$ j.jeee.20180602.12

Received: April 12, 2018; Accepted: June 2, 2018; Published: June 20, 2018

\begin{abstract}
The compressed sensing algorithm based on gradient projection for spare reconstruction (CS-GPSR) is applied to electrical capacitance tomography (ECT) image reconstruction in this paper. First, using the orthogonal basis of FFT transformation, the grey signals of original images can be sparse. Secondly, the observation matrix of ECT system was designed by rearranging the exciting-measuring order, and the capacitance measurements and corresponding sensitivity matrix can be obtained. Finally, the reconstructed images can be obtained using CS-GPSR algorithm. Simulation experiments were carried out and the results showed that the reconstructed images with higher quality can be obtained using the presented CS-GPSR algorithm, compared with conventional linear back projection (LBP) and Landweber iterative algorithms.
\end{abstract}

Keywords: Electrical Capacitance Tomography, Image Reconstruction, Compressed Sensing, Gradient Projection for Sparse Reconstruction

\section{Introduction}

Electrical capacitance tomography (ECT) technique was developed in 1980s, which is widely used for on-line monitoring multi-phase flow in industrial pipeline. ECT technique based on capacitance sensitivity mechanism has many advantages, such as non-radiation, non-intrusion, simple structure, low cost, easy installation and quick response. At present, it has been widely applied in the fields of modern industrial production, such as chemical, petroleum and electric power [1-5].

The schematic diagram of ECT system can be seen in Figure 1.

The process of image reconstruction of the ECT system is as follows: the data acquisition system generates a sinusoidal voltage signal of a certain frequency and transmits it to the exciting electrode of the sensor array. And then, the capacitance values between different electrode pairs are measured. Finally, the cross-sectional permittivity distribution image of media in the pipe can be obtained using some kind of image reconstruction algorithm.

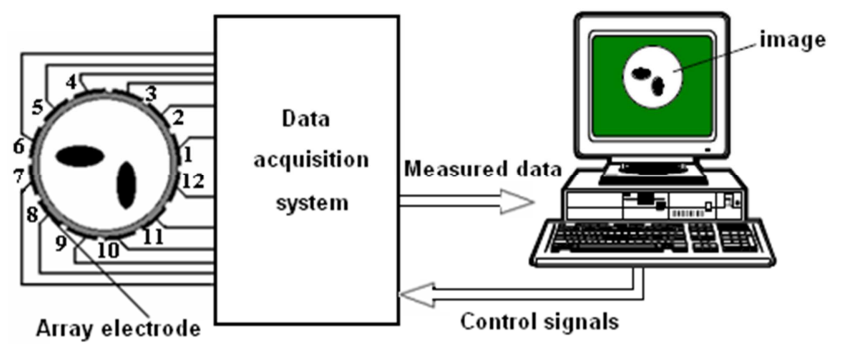

Figure 1. Reconstructed images and binary images of ECT.

ECT image reconstruction is a typical ill-posed problem and its solution is unstable. As a result, the accuracy of image reconstruction algorithms will limit the application of ECT in industrial field. Image algorithms of ECT can be divided into non-iterative and iterative algorithms. Non-iterative algorithm is simple and fast, such as linear back projection (LBP) algorithm, but the quality of the reconstructed image is low, which cannot meet the requirement of industrial 
production. Iterative algorithm, such as Landweber iterative algorithm, has higher precision for image reconstruction. However, its convergence speed is slow and it has the semi-convergence characteristic.

Recently, a variety of algorithms have been studied to improve the visualization quality, which may be summarized as follows:

(1) Research on the reconstruction model of ECT.

The reconstruction model of ECT is naturally nonlinear. Some researchers would like to find some kind of nonlinear reconstruction model or modify the sensitivity matrix to obtain better reconstructed images.

(2) Making full use of priori information. ECT image reconstruction problem is often converted to an optimization problem, in which the priori information can be integrated into the regularization term, and the quality of reconstructed images can be greatly improved. However, the definition of loss function is crucial for this kind of algorithm, which will directly determine the reconstruction results.

(3) Sparse representation based algorithm. In recent years, sparsity regularization has been widely applied to image processing and image reconstruction as the sparsity is one of distinct characteristic of natural images. In 2009, Wright et al represented the test sample based on the overcomplete dictionary with the training samples as the base elements [6]. Ye et al. presented a new basis based on which the permittivity distribution to be reconstructed for ECT is naturally sparse in 2015 [7]. With recent development of compressed sensing (CS), several image reconstruction algorithms for ECT based on CS were proposed, in which the sparsity process is indispensable and the measurement matrix needed to be designed to meet the conditions of CS [8-10]. These will be complicated and time-consuming, and the reconstructed images will be better when the real distribution tends to be sparser.

In 2004, Donoho and Candès proposed compressed sensing (CS) theory [11]. It breaks through the limitation of the traditional sampling theorem on the signal bandwidth and makes the original signal can be precisely reconstructed with a small amount of sampling data, which brings a revolutionary breakthrough for the signal acquisition technology. The theory states that if the signal is sparse or compressible in a certain transform domain, the random observation matrix can be used to obtain the low dimensional measurement signal. By solving an optimization problem, the original signal can be accurately reconstructed from the low dimensional measurement signal. At present, the compressed sensing theory has been widely used in radar, communication, compressed imaging, medical imaging and other fields.

In this paper, the compressed sensing algorithm based on gradient projection for spare reconstruction (CS-GPSR) was applied to ECT image reconstruction. Firstly, the grey signals of original images were transformed into sparse signals by using the Fast Fourier Transformation basis. Secondly, the observation matrix was designed by rearranging the rows of the sensitivity matrix of ECT in a random order and the measurement projection vector was designed by rearranging the rows of the capacitance value vector in the same order. Thirdly, the mathematical model of ECT system was established. Finally, reconstructed images were carried out using LBP, Landweber iterative algorithm and CS-GPSR algorithm, respectively. And also the image binarization of reconstruction images were done based on the optimal threshold method. Experimental results show that the reconstructed images with higher accuracy can be obtained using CS-GPSR algorithm.

\section{Principle of Compressed Sensing}

The premise condition of the compressed sensing theory is that the signal must be sparse or compressible. However, the signals in reality are often not sparse. To solve this problem, an orthogonal transformation is usually applied to sparsely represent the signal and make it satisfy the requirement of CS theory.

For a discrete signal $x$ of length $N$, it can be linearly expressed by a set of $N$ dimensional basis vectors

$$
\mathrm{x}=\sum_{\mathrm{i}=1}^{\mathrm{N}} \psi_{\mathrm{i}} \mathrm{s}_{\mathrm{i}}=\Psi_{\mathrm{S}}
$$

Where $\Psi$ is an orthogonal matrix of $N \times N$, $\Psi_{\mathrm{N} \times \mathrm{N}}=\left[\begin{array}{llll}\psi_{1} & \psi_{2} & \cdots & \psi_{\mathrm{N}}\end{array}\right] . s$ is the coefficient vector of $N \times 1$. If $s$ only has $\mathrm{K}(\mathrm{K}<<\mathrm{N})$ non-zero coefficients, $x$ is sparse under the basis $\Psi$ and its sparse degree is $K$.

Several orthogonal bases can be used to sparsely represent the signal $x$, such as Fast Fourier transform basis (FFT), discrete cosine transform basis (DCT), discrete sine transform basis (DST) and discrete wavelet transform basis (DWT).

The core of the compressed sensing theory is linear measurement. Through designing appropriate linear observation system, $\mathrm{M}(\mathrm{M}<<\mathrm{N})$ measurement values of the original signal are obtained, from which the original signal $x$ of $N \times 1$ can be reconstructed.

$$
\mathrm{y}=\Phi \mathrm{x}=\Phi \Psi \mathrm{s}=\mathrm{A}^{\mathrm{cs}} \mathrm{s}
$$

Where $\mathrm{y}$ is the measurement value vector of $\mathrm{M} \times 1 . \Phi$ is the observation matrix of $\mathrm{M} \times \mathrm{N} \cdot \mathrm{A}^{\mathrm{CS}}\left(\mathrm{A}^{\mathrm{CS}}=\Phi \Psi\right)$ is the compressed sensing matrix of $\mathrm{M} \times \mathrm{N}$.

Reconstructing $x$ from the given $\mathrm{y}$ according to equation (2) is a linear programming problem. Since the number of equations is less than the number of unknowns $(\mathrm{M}<<\mathrm{N})$, equation (2) is ill-conditioned and usually has no definite solution. However, using the prior condition of $K$ sparse, equation (2) has a chance to be solved.

According to compressed sensing theory, when the compressed sensing matrix $\mathrm{A}^{\mathrm{CS}}$ satisfies the restricted isometry property (RIP) criterion, $K$ nonzero coefficients of $s$ can be accurately reconstructed from $M$ observation values by using a variety of CS reconstruction algorithm, while 
ensuring the convergence of the reconstruction process. The equivalent condition of RIP is that the observation matrix $\Phi$ is uncorrelated to sparse matrix $\Psi$. It was already proved that when Gaussian random matrix is used as the observation matrix and arbitrary orthogonal basis is used as the sparse matrix, the compressed sensing matrix $\mathrm{A}^{\mathrm{CS}}$ satisfies the RIP condition under great probability, which means that the ill-conditioned equation (2) can be solved.

The most direct approach to make use of the sparse or compressible prior condition is using the minimum L0 norm model.

$$
\begin{aligned}
& \mathrm{s}_{\mathrm{opt}}=\arg \underset{\mathrm{s}}{\min }\|\mathrm{s}\|_{0} \quad \text { s.t. } \mathrm{y}=\Phi \Psi \mathrm{s} \\
& \mathrm{x}_{\mathrm{opt}}=\Psi \mathrm{s}_{\mathrm{opt}}
\end{aligned}
$$

Because the L0 norm is non-convex, the numerical computation problem of equation (3) is NP-hard and very unstable. Up to now, various alternative models and corresponding CS reconstruction algorithms have been proposed, in which the basis pursuit algorithm, the matching pursuit algorithm, the iterative threshold algorithm and the minimum total variation algorithm are commonly used algorithms.

\section{CS-GPSR Algorithm for ECT}

The Fast Fourier transform (FFT) has a better effect on the binary image signal, which can be used as a sparse basis for ECT system to transform the image gray signal into the sparse signal.

$$
\mathrm{g}=\Psi_{\mathrm{FFT}} \mathrm{S}
$$

where $\mathrm{g}$ is the normalized gray vector of $N \times 1 . \Psi_{\mathrm{FFT}}$ is FFT basis of $N \times N . s$ is the sparse coefficient vector of $\mathrm{N} \times 1$.

The compressed sensing theory requires that the observation process must be linear. The linear model of ECT system is as follows:

$$
\lambda=\mathrm{Sg}
$$

Substituting (4) into (5), the mathematical model of ECT system based on compressed sensing theory can be obtained as expressed in (6)

$$
\lambda=\mathrm{Sg}=\mathrm{S} \Psi_{\mathrm{FFT}} \mathrm{S}=\mathrm{A}^{\mathrm{ECT}} \mathrm{S}
$$

where $\lambda$ is the measurement projection vector of $\mathrm{M} \times 1 . S$ of $\mathrm{M} \times \mathrm{N}$ is the sensitivity matrix of ECT system and observation matrix of CS. $g$ is grey vector of $N \times 1 \cdot A^{E C T}$ is the compressed sensing matrix of ECT system.

The number of the independent capacitance measurement values which are acquired by the 16-electrode ECT system is $M=16 \times 15 / 2=120$. In order to ensure the imaging accuracy of the ECT system, the image area for the reconstructed image is divided into $32 \times 32$ square grids and the number of pixels in the imaging area of pipe is 812. According to compressed sensing theory, under the condition that when the compressed

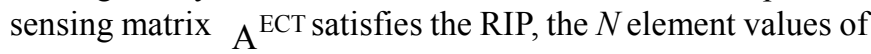
the image grey vector $g$ can be reconstructed accurately from the $M$ independent capacitance measurements of the projection vector $\lambda$, that is to say, the observation matrix must be Gaussian random matrix.

However, due to the limitation of the ECT system hardware and inherent sampling method, it is very difficult to realize random observation and acquire the corresponding capacitance measurement data. In order to improve the randomness of the observation matrix, in this paper, the random observation matrix was designed by rearranging the rows of the sensitivity matrix $S$ in a random order and the corresponding random measurement projection vector was obtained by rearranging the rows of the capacitance value vector $\lambda$ in the same order. By doing that, the random observation matrix is uncorrelated to sparse matrix, which satisfies the RIP under a certain probability.

In reference [12], it was pointed out that when the observation matrix and sparse matrix are not related, the minimum L1 norm problem and the minimum L0 norm problem have the same solution. The minimum L1 norm problem is a convex optimization problem, which can be easily transformed into a linear programming problem.

$$
\begin{aligned}
& \mathrm{s}_{\text {opt }}=\arg \underset{\mathrm{s}}{\min }\|\mathrm{s}\|_{1} \quad \text { s.t. } \quad \lambda_{\text {new }}=\mathrm{S}_{\text {new }} \Psi_{\mathrm{FFT}} \mathrm{S} \\
& \mathrm{g}_{\mathrm{opt}}=\Psi_{\mathrm{FFT}} \mathrm{s}_{\text {opt }}
\end{aligned}
$$

In order to solve equation (7), the constrained convex optimization problem is transformed into an unconstrained convex optimization problem. Meanwhile, the L1 norm regularization model can be used to overcome the ill-condition of the ECT system.

$$
\begin{aligned}
& \mathrm{s}_{\mathrm{opt}}=\underset{\mathrm{s}}{\arg \min }\left\{\frac{1}{2}\left\|\mathrm{~S}_{\text {new }} \Psi_{\mathrm{FFT}} \mathrm{s}-\lambda_{\text {new }}\right\|_{2}^{2}+\tau\|\mathrm{s}\|_{1}\right\} \\
& \mathrm{g}_{\mathrm{opt}}=\Psi_{\mathrm{FFT}} \mathrm{s}_{\mathrm{opt}}
\end{aligned}
$$

In this paper, the gradient projection for spare reconstruction algorithm based on compressed sensing theory (CS-GPSR) is used to solve the model expressed in equation (8) and to realize the image reconstruction of ECT system.

The GPSR algorithm is a classical algorithm for the signal reconstruction of CS [13]. By splitting the variable s into its positive and negative parts (i.e. $\mathrm{s}=\mathrm{u}-\mathrm{v}$, where $\left.\mathrm{u}=(\mathrm{s})_{+}=\max (0, \mathrm{~s}) \geq 0, \quad \mathrm{v}=(-\mathrm{s})_{+} \geq 0\right)$, the equation (9) of the L1 norm regularization model can be transformed into the quadratic programming problem which is expressed in equation (10).

$$
\min _{\mathrm{s}} \frac{1}{2}\|\lambda-\mathrm{As}\|_{2}^{2}+\tau\|\mathrm{s}\|_{1}
$$




$$
\min _{\mathrm{u}, \mathrm{v}} \frac{1}{2}\|\lambda-\mathrm{A}(\mathrm{u}-\mathrm{v})\|_{2}^{2}+\tau \mathrm{l}_{\mathrm{N}}^{\mathrm{T}} \mathrm{u}+\tau \mathrm{l}_{\mathrm{N}}^{\mathrm{T}} \mathrm{v}
$$

where $1_{\mathrm{N}}=[1,1, \cdots, 1]^{\mathrm{T}}$ is the column vector consisting of $N$ ones. After deduction, problem (10) can be rewritten as more standard quadratic programming form as showed in equation (11).

$$
\begin{aligned}
& \min c^{T} z+\frac{1}{2} z^{T} B z \equiv F(z) \\
& \text { s.t. } \quad z \geq 0
\end{aligned}
$$

where $\quad \mathrm{z}=\left[\begin{array}{l}\mathrm{u} \\ \mathrm{v}\end{array}\right], \quad \mathrm{b}=\mathrm{A}^{\mathrm{T}} \lambda \quad, \quad \mathrm{c}=\tau 1_{2 \mathrm{~N}}+\left[\begin{array}{c}-\mathrm{b} \\ \mathrm{b}\end{array}\right] \quad$ and $\mathrm{B}=\left[\begin{array}{cc}\mathrm{A}^{\mathrm{T}} \mathrm{A} & -\mathrm{A}^{\mathrm{T}} \mathrm{A} \\ -\mathrm{A}^{\mathrm{T}} \mathrm{A} & \mathrm{A}^{\mathrm{T}} \mathrm{A}\end{array}\right]$.

The GPSR algorithm bases on the steepest descent direction $-g^{(i)}$ and uses Armijo rule performing a backtracking line search to update the iteration step $\alpha_{0}$.

Where $\mathrm{g}_{\mathrm{k}}^{(\mathrm{i})}=\left\{\begin{array}{ll}\left(\nabla \mathrm{F}\left(\mathrm{z}^{(\mathrm{i})}\right)\right)_{\mathrm{k}}, & \mathrm{z}^{(\mathrm{i})}>0 \quad \text { or } \quad\left(\nabla \mathrm{F}\left(\mathrm{z}^{(\mathrm{i})}\right)\right)_{\mathrm{k}}<0 \\ 0, & \text { otherwise }\end{array}\right.$.

Along the negative gradient direction $-g^{(i)}$, the steepest descent step $\alpha_{0}$ for each update is satisfied, which can be computed explicitly as

$$
\alpha_{0}=\frac{\left(g^{(i)}\right)^{T} g^{(i)}}{\left(g^{(i)}\right)^{T} \mathrm{Bg}^{(i)}}
$$

The iterative procedure of GPSR algorithm is as follows:

Initialization: select initial value of $\mathrm{z}^{(0)}$, and choose parameter $\beta \in(0,1)$ and $\mu \in(0,1 / 2)$, and set $i=0$.

Step 1: compute $\alpha_{0}$ according the equation (12).

Step 2: select $\alpha^{(i)}$ from sequence $\alpha_{0}, \beta \alpha_{0}, \beta^{2} \alpha_{0}, \ldots \ldots$ in turn, until the following condition is met $\mathrm{F}\left(\mathrm{z}^{(\mathrm{i}+1)}\right) \leq \mathrm{F}\left(\mathrm{z}^{(\mathrm{i})}\right)-\mu \nabla \mathrm{F}\left(\mathrm{z}^{(\mathrm{i})}\right)^{\mathrm{T}}\left(\mathrm{z}^{(\mathrm{i})}-\mathrm{z}^{(\mathrm{i}+1)}\right) \quad, \quad$ where $\mathrm{z}^{(\mathrm{i}+1)}=\left(\mathrm{z}^{(\mathrm{i})}-\alpha^{(\mathrm{i})} \nabla \mathrm{F}\left(\mathrm{z}^{(\mathrm{i})}\right)\right)_{+}$

Step 3: Check whether the termination condition is satisfied.

If the termination condition expressed in equation (13) is satisfied, then $\mathrm{z}^{(\mathrm{i}+1)}$ will be output. Otherwise, return back to step 1 .

$$
\left|\frac{F\left(z^{(i+1)}\right)-F\left(z^{(i)}\right)}{F\left(z^{(i)}\right)}\right| \leq \text { tolp }
$$

The grey values of the reconstructed images are not binary. In order to compare the quality of reconstructed images obtained by LBP, Landweber and CS-GPSR algorithms, the optimal threshold method was used to binarize the initial reconstructed image. The optimal threshold is determined by considering all possible thresholds and choosing the threshold which has the minimum error from the measured values, which can be expressed as follows:

$$
\min \left\|\operatorname{Sg}^{\text {th }}-\lambda\right\|_{2}^{2}
$$

where $\mathrm{g}^{\text {th }}$ is the gray value vector of the image obtained by the optimal threshold method.

\section{Simulation Experiment}

In this paper, four kinds of typical flow patterns were set to simulate the oil-gas two-phase flow. The relative permittivity of oil and gas were set to be 3 and 1, respectively. COMSOL 3.5a (finite element analysis software) was used to create ECT sensor simulation model and calculate the simulated capacitance measurements. MATLAB software was used to develop the ECT image reconstruction algorithm and image processing. The imaging area of pipe is divided into 812 pixels by square grid.

In the simulation process, the initial images were reconstructed by LBP algorithm, Landweber iterative algorithm and the CS-GPSR algorithm, which can be seen in Figure 2 (a). After that, the optimal threshold method was used to obtain binary images, which can be shown in Figure 2 (b).

From Figure 2 (a), it can be seen that the quality of the reconstructed images by the CS-GPSR algorithm is obviously improved compared with that of LBP algorithm and Landweber iterative algorithm. The CS-GPSR algorithm can clearly reconstruct the location information and edge information of the objects, that is to say, the object reconstructed by the CS-GPSR algorithm has an accurate distribution, a regular edge and a high shape fidelity. On the contrary, the object reconstructed by LBP algorithm and Landweber iterative algorithm has many obvious artifacts, which make the edge of object indistinguishable. From the reconstructed image of the flow patterns 3 and 4, it can be seen that for relatively complex flow patterns, the image reconstructed by LBP algorithm is completely distorted and the image accuracy reconstructed by Landweber iterative algorithm is also low, however, the reconstructed images with good accuracy and shape fidelity can also be obtained using CS-GPSR algorithm.

It can also be seen from Figure $2(b)$, after optimal threshold processing, the reconstructed images by CS-GPSR algorithm are more close to the original distribution and have no artifact. The shape fidelity of CS-GPSR is best for the studied three algorithms. 


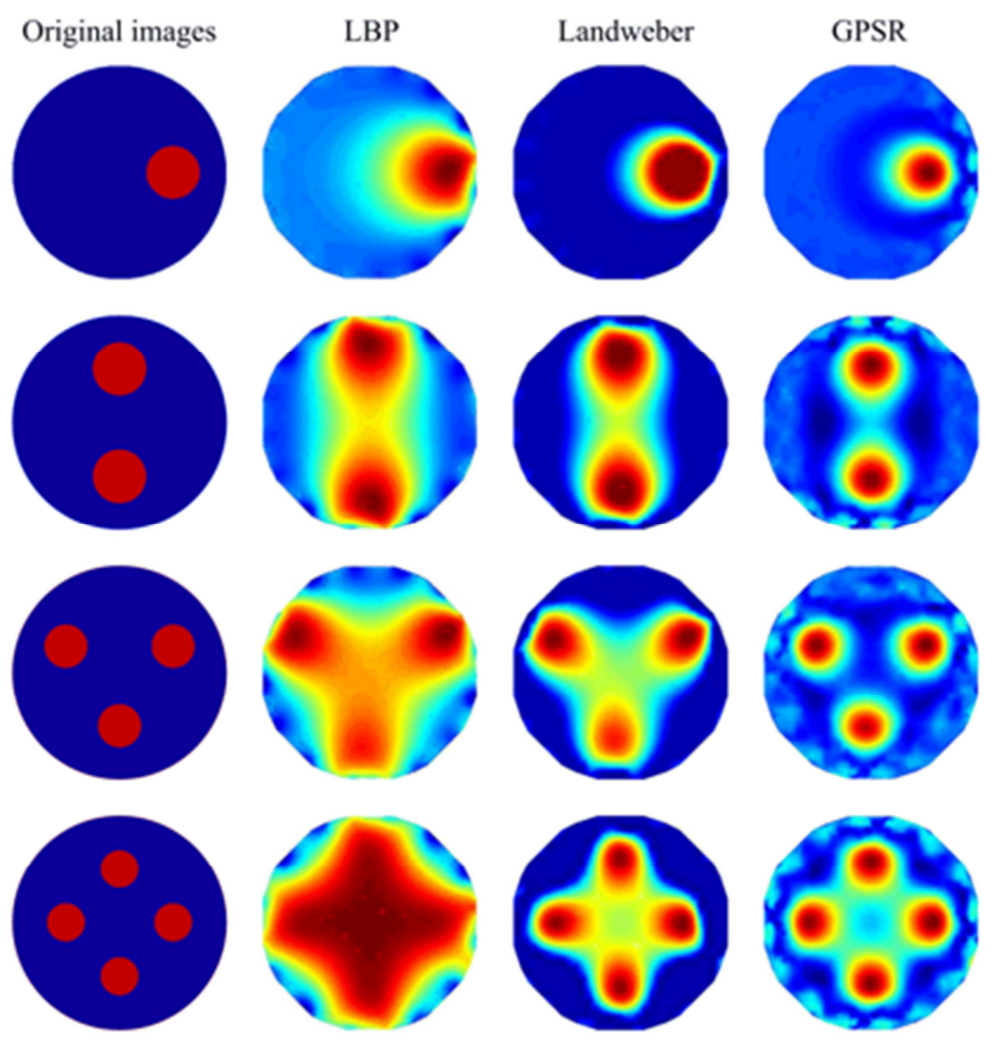

(a) Reconstructed images

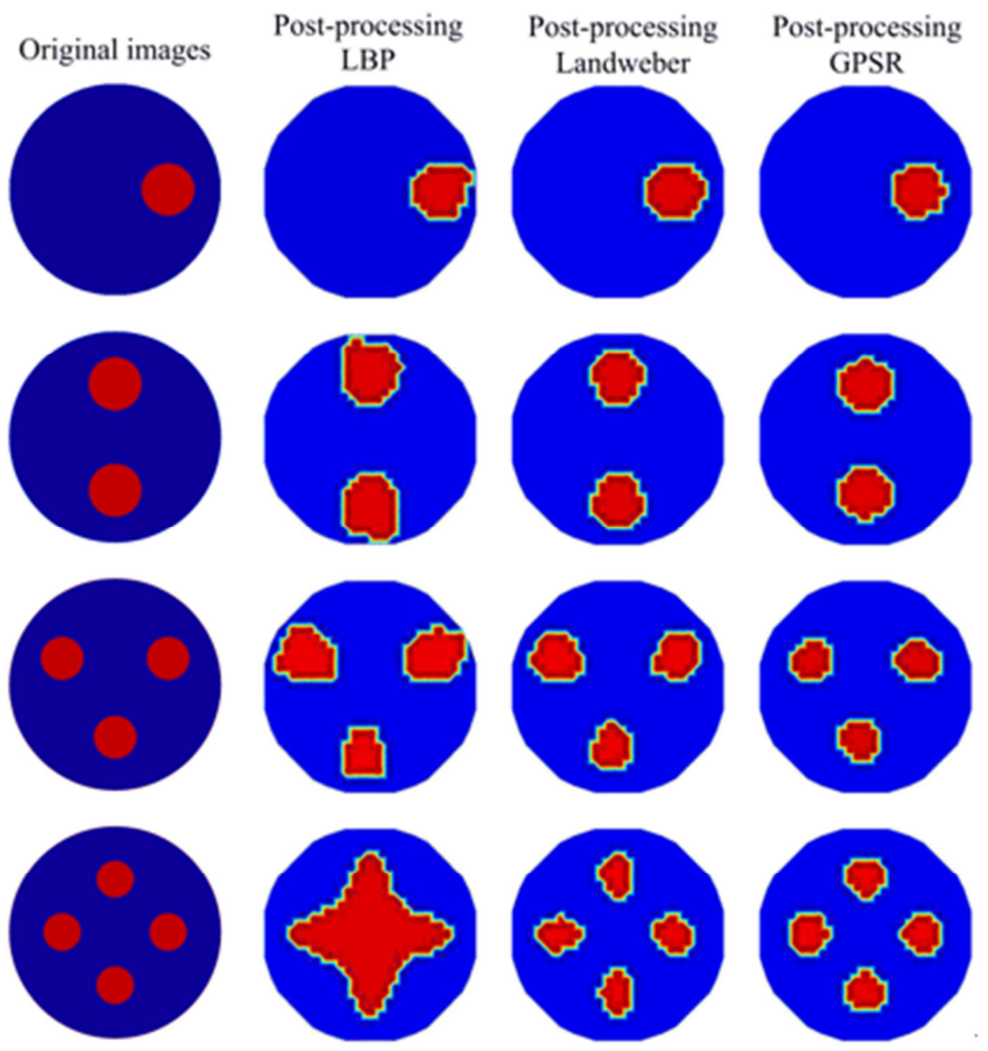

(b) Binary images

Figure 2. Reconstructed images and binary images of ECT.

In order to quantitatively evaluate the performance of this algorithm, the relative error $(R E)$ and the correlation coefficient $(C C)$ were used as the evaluating index for the reconstruction algorithm, which are defined as follows [14]: 


$$
\begin{aligned}
& R E=\frac{\|\widehat{g}-g\|_{2}}{\|g\|_{2}} \\
& C C=\frac{\sqrt{\sum_{i=1}^{N}\left(g_{i}-\bar{g}\right)\left(\overline{\left.g_{i}-g\right)}\right.}}{\sqrt{\sum_{i=1}^{N}\left(g_{i}-\bar{g}\right)^{2} \cdot \sum_{i=1}^{M}\left(\overline{\left.g_{i}-g\right)^{2}}\right.}}
\end{aligned}
$$

where $g$ and $\hat{g}$ are the true permittivity distribution of the test object and the reconstructed permittivity distribution, respectively. $\bar{g}$ and $\bar{g}$ are the mean values of $g$ and $\bar{g}$, respectively. $N$ is the number of the pixels in the imaging area and $N$ equals 812 in our simulation.

The relative error $(R E)$ and the correlation coefficient $(C C)$ for the reconstructed images in Figure $2(a)$ and $(b)$ were calculated and were listed in Tables 1 to 4 .

Table 1. Calculated RE of reconstructed images in Figure 2 (a).

\begin{tabular}{llll}
\hline Flow pattern & LBP & Landweber & CS-GPSR \\
\hline Pattern 1 & 1.2383 & 0.8081 & 0.6768 \\
Pattern 2 & 1.5709 & 0.9289 & 0.7451 \\
Pattern 3 & 2.0027 & 1.0993 & 0.9793 \\
Pattern 4 & 2.0528 & 1.1262 & 1.1019 \\
\hline
\end{tabular}

Table 2. Calculated RE of reconstructed images in Figure 2 (b).

\begin{tabular}{llll}
\hline Flow pattern & $\begin{array}{l}\text { Post-processing } \\
\text { LBP }\end{array}$ & $\begin{array}{l}\text { Post-processing } \\
\text { Landweber }\end{array}$ & $\begin{array}{l}\text { Post-processing } \\
\text { CS-GPSR }\end{array}$ \\
\hline Pattern 1 & 0.7845 & 0.3922 & 0.1961 \\
Pattern 2 & 0.8720 & 0.6124 & 0.2887 \\
Pattern 3 & 1.2583 & 0.6697 & 0.4208 \\
Pattern 4 & 1.0308 & 0.6892 & 0.5590 \\
\hline
\end{tabular}

Table 3. Calculated CC of reconstructed images in Figure 2 (a).

\begin{tabular}{llll}
\hline Flow pattern & LBP & Landweber & CS-GPSR \\
\hline Pattern 1 & 0.5693 & 0.7514 & 0.8397 \\
Pattern 2 & 0.4458 & 0.6069 & 0.8394 \\
Pattern 3 & 0.3578 & 0.5811 & 0.7149 \\
Pattern 4 & 0.3228 & 0.5973 & 0.6321 \\
\hline
\end{tabular}

Table 4. Calculated CC of reconstructed images in Figure 2 (b).

\begin{tabular}{llll}
\hline Flow pattern & $\begin{array}{l}\text { Post-processing } \\
\text { LBP }\end{array}$ & $\begin{array}{l}\text { Post-processing } \\
\text { Landweber }\end{array}$ & $\begin{array}{l}\text { Post-processing } \\
\text { CS-GPSR }\end{array}$ \\
\hline Pattern 1 & 0.6966 & 0.9204 & 0.9778 \\
Pattern 2 & 0.6819 & 0.8087 & 0.9522 \\
Pattern 3 & 0.5522 & 0.7893 & 0.9107 \\
Pattern 4 & 0.5002 & 0.7765 & 0.8993 \\
\hline
\end{tabular}

For the evaluating index $R E$ and $C C$, it can be seen from their definition in equations (15) and (16) that smaller RE and larger CC imply the reconstructed images with higher quality for ECT. Through analyzing the evaluating index $R E$ and $C C$ in Tables 1 to 4 , it can be seen that the relative error $(R E)$ of the reconstructed images using CS-GPSR algorithm is the smallest compared that of LBP and Landweber iterative algorithms. Meanwhile, the correlation coefficient $(C C)$ of the reconstructed images using GPSR algorithm is the largest compared with that of LBP and Landweber iterative algorithm, which shows that the reconstructed images based on CS-GPSR are more close to the real distribution.

After the optimal threshold method processing, the accuracy of the three algorithms are improved obviously, but the reconstruction effect of the CS-GPSR algorithm is better than the other two algorithms. Therefore, the algorithm discussed in this paper can be applied to the ECT image reconstruction, by using which the reconstructed images with higher accuracy can be obtained.

\section{Conclusion}

In this paper, the compressed sensing algorithm based on gradient projection for spare reconstruction (CS-GPSR) was applied to ECT image reconstruction. Because the CS theory can accurately reconstruct the original signal with less observation data, it is effective to solve the ill-conditioned problem of ECT image reconstruction. Meanwhile, the CS-GPSR algorithm can effectively reconstruct the location information and edge information of the object in the field. Simulation results show that the proposed algorithm can reconstruct a high precision images with less observation data, which provides a new way and method for ECT image reconstruction.

\section{Acknowledgements}

The authors thank the National Natural Science Foundation of China (No. 51306058) and the Fundamental Research Funds for the Central Universities (No. 2017MS131) for supporting this research. Dr. Lifeng Zhang would also like to thank the China Scholarship Council for supporting his visit to the University of Manchester.

\section{References}

[1] H. S. Tapp, A. J. Peyton, E. K. Kemsley and R. H. Wilson, "Chemical engineering applications of electrical process tomography," Sensors Actuat. B-Chem., 2003, vol. 92 pp. 1724.

[2] I. Ismail, J. C. Gamio, S. F. A. Bukhari, and W. Q. Yang, "Tomography for multi-phase flow measurement in the oil industry," Flow Meas. Instrum., 2005, vol. 16 pp. 145-155.

[3] H. X. Wang and L. F. Zhang, "Identification of two-phase flow regimes based on support vector machine and electrical capacitance tomography," Meas. Sci. Technol., 2009, vol. 20 pp. 114007.

[4] G. McKenzie and P. Record, "Prognostic monitoring of aircraft wiring using electrical capacitive tomography," Rev. Sci. Instrum., 2011, vol. 82 pp. 124705.

[5] W. Q. Yang and S. Liu, "Role of tomography in gas/solids flow measurement," Flow Meas. Instrum., 2000, vol. 11 pp. 237244.

[6] J. Wright, A. Y. Yang, A. Ganesh, S. S. Sastry, and Y. Ma, "Robust face recognition via sparse representation," IEEE Trans. Pattern Anal. Mach. Intell., 2009, vol. 31 pp. 210-227. 
[7] J. M. Ye, H. G. Wang, and W. Q. Yang a, "Image reconstruction for electrical capacitance tomography based on sparse representation," IEEE T. Instrum. Meas., 2015, vol. 64 pp. 89 102.

[8] H. C. Wang, I. Fedchenia, S. L. Shishkin, A. Finn, L. L. Smith, and M. Colket, "Sparsity-inspired image reconstruction for electrical capacitance tomography," Flow Meas. Instrum., 2015, vol. 43 pp. 59-71.

[9] L. F. Zhang, Z. L. Liu, and P. Tian, "Image reconstruction algorithm for electrical capacitance tomography based on compressed sensing," Acta Electronica Sinica, 2017, vol. 45 pp. 353-358.

[10] W. Q. Yang, D. M. Spink, T. A. York, and H. McCann, "Optimization of an iterative image reconstruction algorithm for electrical capacitance tomography," Meas. Sci. Technol., 1999, vol. 10 pp. L37-L39.

[11] D. L. Donoho, "Compressed sensing," IEEE T. Inform. Theory, 2006, vol. 52 pp. 1289-1306.

[12] S. S. Chen, D. L. Donoho, M. A. Saunders, "Atomic decomposition by basis pursuit," SIAM J. Sci. Comput., 2001, vol. 43 pp. $129-159$.

[13] M. A. T Figueiredo, R. D Nowak, S. J. Wright, "Gradient Projection for Sparse Reconstruction : Application to Compressed Sensing and Other Inverse Problem," IEEE J-STSP, 2007, vol. 1 pp. 586-597. 